\title{
Novel Leak Localization in Pressurized Pipeline Networks using Acoustic Emission and Geometric Connectivity
}

Didem Ozevin ${ }^{1,1}$ and James Harding ${ }^{1}$

${ }^{1}$ Department of Civil and Materials Engineering, University of Illinois at Chicago, Chicago, IL 60607

Time dependent aging and instantaneous threats can cause the initiation of damage in the buried and on-ground pipelines. Damage may propagate all through the structural thickness and cause leaking. The leakage detection in oil, water, gas or steam pipeline networks before it becomes structurally instable is important to prevent any catastrophic failures. The leak in pressurized pipelines causes turbulent flow at its location, which generates solid particles or gas bubbles impacting on the pipeline material. The impact energy causes propagating elastic waves that can be detected by the sensors mounted on the pipeline. The method is called Acoustic Emission, which can be used for real time detection of damage caused by unintentional or intentional sources in the pipeline networks. In this paper, a new leak localization approach is proposed for pipeline networks spread in a two dimensional configuration. The approach is to determine arrival time differences using cross correlation function, and introduce the geometric connectivity in order to identify the path that the leak waves should propagate to reach the AE sensors. The leak location in multi-dimensional space is identified in an effective approach using an array of sensors spread on the pipeline network. The approach is successfully demonstrated on laboratory scale polypropylene pipeline networks.

Keywords: Pipeline network, leak, acoustic emission, geometric connectivity

\section{Introduction}

Pipeline networks are regularly inspected using methods such as smart pigs that are cylindershaped electronic devices to detect metal loses, mapping tools based on GPS for above ground pipelines, guided wave ultrasonics and hydrostatic testing [1]. Rose et al. [2] are the pioneers in the use of guided wave ultrasonic method to inspect pipelines, and they discuss the latest generation guided wave approach for long range pipeline inspection. Elliott et al. [3] developed "SmartBall” which is a spherical acoustic device travelling through the pipeline for leak detection. The inspection methods are applied based on the maintenance schedule of pipelines. They cannot detect instantaneous changes in the structure due to sources such as impact, crack growth. Caleyo et al. [4] demonstrated that prioritizing the pipeline inspection and maintenance based on the failure data might establish incorrect prioritization due to significant uncertainty of pooling failure of dissimilar pipeline systems data.

As continuous online monitoring methods, Kishawy and Gabbar [1] list the conventional monitoring methods for leak detection such as mass-balance method, pressure drop method, and consider these methods as the laborious and inefficient, and conclude that the pipeline integrity technologies must continue to evolve. As a real time monitoring system, Shinozuka et al. [5] developed wireless MEMS based acceleration sensor (frequency in the range of $\mathrm{Hz}$ ) networks for monitoring damage in water pipeline networks. Wan et al. [6] developed an automated pipeline monitoring system for detecting cutters as potential threats to pipelines. The system uses audible

\footnotetext{
${ }^{1}$ Corresponding author, Phone: 1-312-413-3051, Fax: 1-312-996-2426, Email: dozevin@uic.edu, Address: 842 W Taylor Street, ERF 3073, Chicago, IL 60607
} 
acoustic waves and a pattern recognition algorithm to differentiate cutter noise and others. Higgins and Paulson [7] implemented the use of fiber optic sensor for Acoustic Emission sensing for detecting wire breaks in concrete pipelines. Inaudi and Glisic [8] presented the use of fiber optic sensor to monitor temperature and strain as an indirect way of leakage. The authors claim that a single fiber optic instrument can monitor up to $60 \mathrm{~km}$.

As discussed above, there are various studies in the literature on sensor development using MEMS and fiber optic technologies for damage detection in pipelines using acoustic methods. In this paper, a new source location methodology is tested on a pipeline network in order to locate the leak source in 2D using 1D source location algorithm and geometric connectivity. The methodology is applicable to any types of novel acoustic sensors.

\section{Acoustic Emission Method}

The Acoustic Emission method is a nondestructive testing method that relies on propagating transient waves generated by sudden stress-strain change in a material such as crack growth, leak, impact. There are two types of acoustic emissions as defined by ASTM E 1316 [9]: burst type as a qualitative description of the discrete signal related to an individual emission event occurring within the material, and continuous type as a qualitative description of the sustained signal level produced by rapidly occurring acoustic emission sources. Majeed and Murthy [10] defined the AE signals including nonzero rise time as compared to traditional zero rise time signal presentation. In this study, the burst type AE signal $V_{\text {burst }}(t)$ for single frequency $f_{o}$ is idealized in order to include the arrival time factor into the formulation using the following equation:

$$
V_{\text {burst }}(t)=V_{o} \sin \left(2 \pi f_{o} t\right)\left\{\left(1-e^{-\left(t-t_{\text {arrival }}\right) / t_{\text {rise }}}\right) \in 0 . .1\right\} e^{-\left(t-t_{\text {arrival }}\right) / t_{\text {decay }}} H\left[t-t_{\text {arrival }}\right]
$$

where the term $\left\{\left(1-e^{-\left(t-t_{\text {arrival }}\right) / t_{\text {rise }}}\right) \in 0 . .1\right\}$ indicates the rise time function normalized to be in the range of 0 to $1, t_{\text {rise }}$ is rise time, the term $e^{-t / t_{\text {decay }}}$ indicates the decay time with $t_{\text {decay }}$, the term $H\left[t-t_{\text {arrival }}\right]$ is Heaviside function indicating the waveform arrival to the sensor at $t_{\text {arrival }}$.

Examples of burst type include crack growth, impact, which are instantaneous sources as shown in Figure 1a based on equation 1 for $100 \mathrm{kHz}$ frequency, $40 \mu \mathrm{sec}$ arrival time, $20 \mu \mathrm{sec}$ rise time and $40 \mu$ sec decay time. The continuous type AE signal $V_{\text {continuous }}(t)$ for single frequency $f_{o}$ is idealized using the following equation:

$$
V_{\text {continuous }}(t)=\sin \left(2 \pi f_{o} t\right) \sum_{i=1}^{\infty} V_{i}\left\{\left(1-e^{-\left(t-t_{\text {arrival }}\right) / t_{\text {rise }}}\right) \in 0 . .1\right\} e^{-\left(t-t_{\text {arrival }}(i)\right) / t_{\text {decay }}(i)} H\left[t-t_{\text {arrival }}(i)\right]
$$

Examples of continuous type include leak, friction which are spatially stationary sources as shown in Figure 1b based on equation 2. As compared to the burst type AE signal, the continuous AE signal does not have a definite rise time, and can be idealized as the summation of multiple wave arrivals using Heaviside functions. The AE patterns of different sources can be used in source discrimination algorithms.

The AE method has been successfully applied to monitor crack growth in pressure vessel steels [11], and assess the structural integrity of various pressurized components [12]. Miller et al. [13] studied the leak rate of $0.38 \mathrm{dm}^{3} / \mathrm{h}$ generated in flange gaskets and pipe threads using the AE method, and showed that below $689 \mathrm{kPa}$ the leak at this rate may not be turbulent so the flow of 
the liquid produces little or no AE. Kosel et al. [14] showed the combination of cross correlation function with an appropriate bandpass filter for locating continuous AE sources.
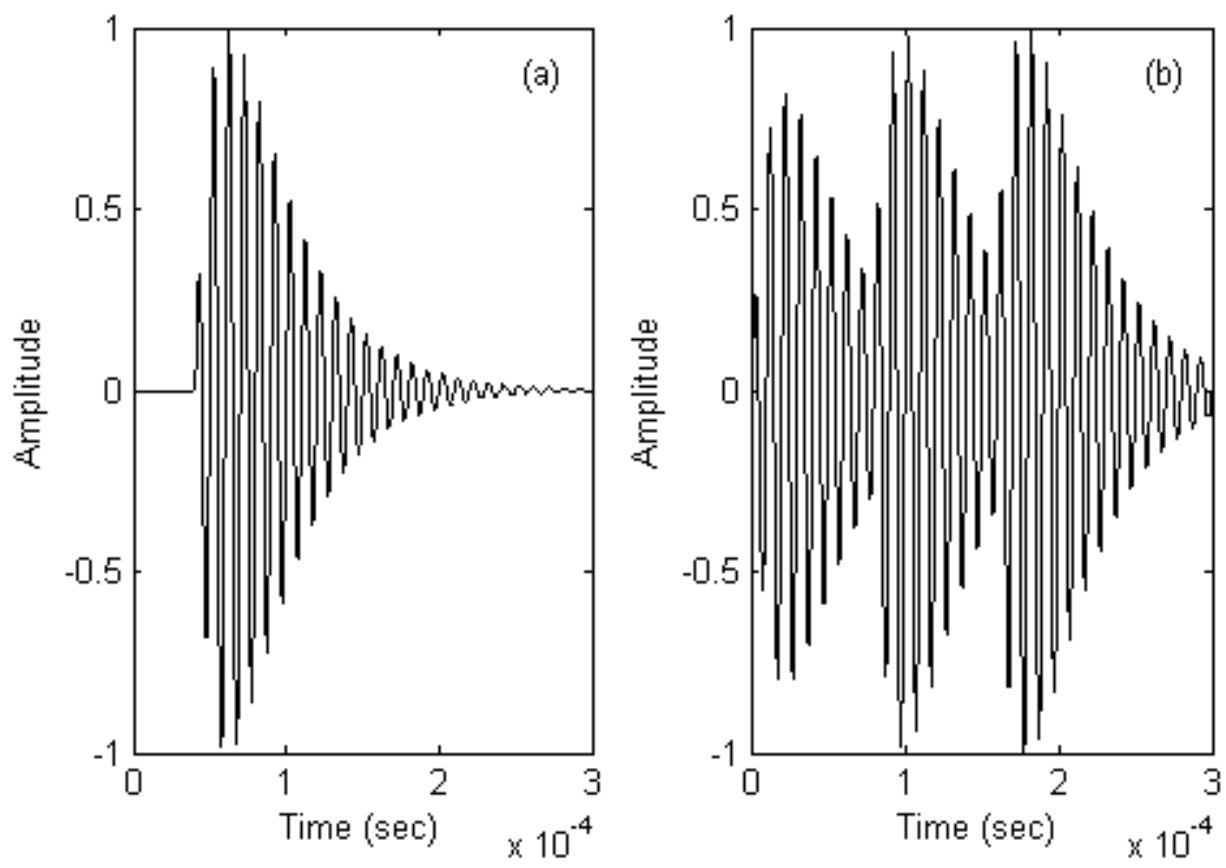

Figure 1- Simulated AE signatures, (a) burst type, (b) continuous type

The AE method is capable of source localization by means of local, global, remote and online monitoring. The ability of the AE method to locate time dependent (e.g. crack growth) or instantaneous sources (e.g. impact) with the sensors further from the flaws is a major advantage as compared to other NDE methods. However, the complexity of the pipeline networks makes the applications of AE method for the source localization using conventional source location methods difficult. Typical assumptions of the source location algorithms are: (a) the AE event originates from a point source, (b) the source to the sensor path is straight, (c) the medium is isotropic and (d) a set of acoustic arrivals is related to a single source [15]. The second assumption causes significant error if the conventional source localization algorithm is applied to structures that are spread in two dimensions while they could be idealized as one dimensional due to the length to cross section ratio.

\section{Theory of Leak Location Methodology}

The location methodology used in this study is based on the source location algorithm developed by Ozevin [16] for truss bridges. The methodology combines the geometric boundaries of the structure to define the shortest wave paths from the leak sources to the AE sensors using the local coordinate system. For example, Figure 2 shows a 2D pipeline network formed by six elements and six joints with a leak source at joint J2. Three AE sensors are mounted on the pipe surface. When leak occurs, it causes turbulence in its vicinity, which propagates through material or liquid for liquid filled pipelines. Propagating waves follow the paths as shown in the figure, and they are detected by the AE sensors. For the given example, the propagating waves reach the AE-1 first and then AE-2 and AE-3, which give the hit sequence. Arrival times for source localization are calculated using the cross correlation approach as discussed in the next section. Using the arrival time differences of sensors $1\left(t_{s 1}\right)$ and $2\left(t_{s 2}\right)$ in the hit sequence, the source 
location $x$ in the local coordinate system is:

$$
x=\frac{\left(t_{s_{1}}-t_{s_{2}}\right) V+L_{s_{1}-s_{2}}}{2}
$$

where $L_{s_{1}-s_{2}}$ is the distance between the first and second hit sensors calculated using the connectivity matrix $\boldsymbol{C}$, joint-sensor matrix $\boldsymbol{M}$, sensor coordinates $\boldsymbol{S}$, and joint coordinates $\boldsymbol{J}$. The connectivity matrix $\boldsymbol{C}$ defines the joints at the vicinity of AE sensors ( 1 if the joint at the ends of the pipeline that the AE sensor is mounted; 0 otherwise as shown in Figure 2). The joint-sensor matrix $\boldsymbol{M}$ defines the joint numbers at the end of each sensor. The connectivity matrix is extracted using the joint-sensor matrix. In the equation, $V$ is wave velocity, which may cause location error in dispersive media such as polymer based pipelines as discussed in Section 5.2. The local acoustic source coordinate $x$ is converted to the global coordinate $X, Y$ and $\mathrm{Z}$ using the following equation:

$$
\left\{\begin{array}{l}
X \\
Y \\
Z
\end{array}\right\}=X\left\{\begin{array}{ll}
\cos \theta_{<X Y, Z>} & \cos \theta_{<X, Y>} \\
\cos \theta_{<X Y, Z>} & \sin \theta_{<X, Y>} \\
\cos \theta_{<X Z, Y>} & \cos \theta_{<Z, X>}
\end{array}\right\}+\left\{\begin{array}{l}
X_{1 h} \\
Y_{1 h} \\
Z_{1 h}
\end{array}\right\}
$$

where $\theta$ is the angle between the structural member that the first hit sensor is mounted on and the global abscissa. The subscript ' $1 h$ ' indicates the coordinates of the first hit sensor. If the geometry is 2D, the last rows of the matrices are eliminated.

For the proposed location algorithm, assuming that waves can propagate from one end of the pipe to the other, if there are $n$ number of pipelines connected each other, number of sensors required for $2 \mathrm{D}$ source location is $n / 2$. A single sensor is adequate to monitor each pipeline joint so that there will be no similar arrival time differences due to leak source anywhere in the network that could cause mislocation. AE source energy due to the target leak rate should be identified in correlation with the attenuation characteristics of material in order to validate the claim that the AE source can propagate along the pipes above the background noise so that two nearest sensors can detect the source. For liquid filled pipelines, the acoustic waves are transmitted by liquid which is less attenuative than the waves propagating in a material. However, for gas filled pipelines, the AE method relies on the propagating elastic waves through material. If the material is highly viscous (e.g. polymer based), the attenuation coefficient measured as loss in $\mathrm{dB} / \mathrm{m}$ is high. As attenuation is proportional with frequency [17], low frequency AE sensors $(<20 \mathrm{kHz})$ can be implemented for such cases. 


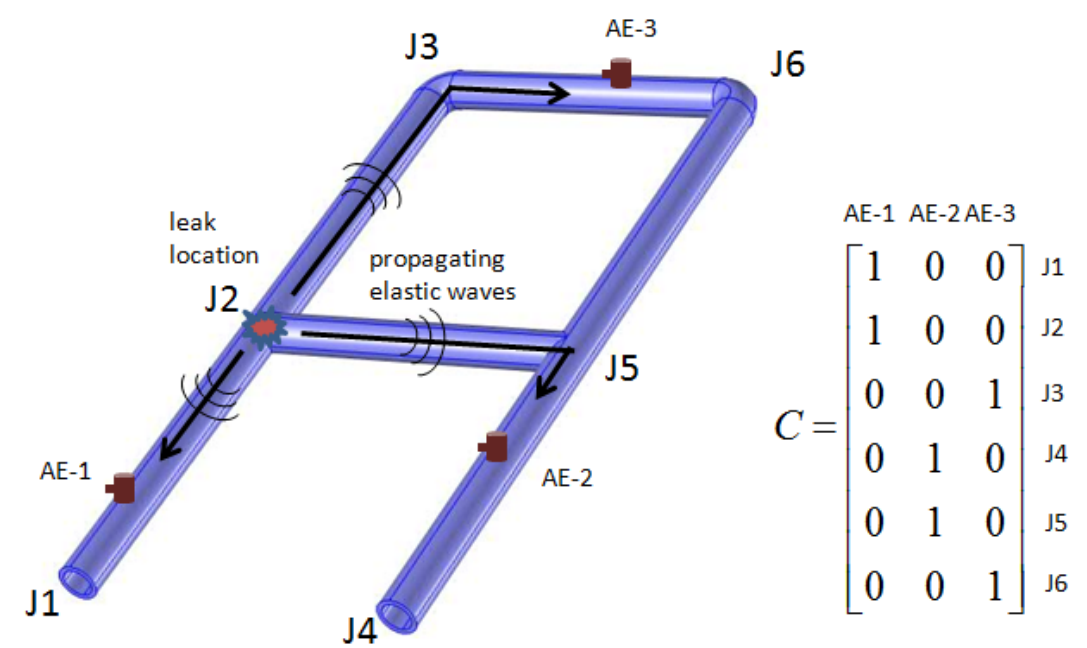

Figure 2 - Leak source at joint J2 in a pipeline network and the joint-sensor connectivity matrix

\section{Cross Correlation Function}

Grabec [18] showed the application of cross correlation method for determining arrival times of continuous waveforms. The cross correlation function for discrete and finite duration signals is

$$
R_{y_{1} y_{2}}(\tau)=\sum_{t=1}^{N} y_{1}(t) y_{2}(t+\tau)
$$

where $R_{y_{1} y_{2}}(\tau)$ is the cross correlation coefficient of two signals, $y_{1}$ and $y_{2}$, as a function of a time delay $\tau, N$ is the lengths of signals. In signal processing, the cross correlation function reveals the degree of similarity between two signals as a function of a time delay [19]. A distinct peak means that two signals are matched for that particular time delay. The method fails if multiple flaw sources are simultaneously active [20]. Grabec [18] emphasizes that undamped behavior of high sensitive AE sensors can cause uncertainty in the arrival time calculation due to long ringing times. Gao et al. [21] studied the effect of background noise and dispersion on the cross correlation result of acoustic waves (about $200 \mathrm{~Hz}$ ). The authors controlled the cut-off frequency of the high pass filter to minimize the interference of background noise. Jiao et al. [22] developed modal based AE approach for the leak location, which require the separation of two dispersive modes and the dispersion model of the pipe. Secondary information is needed for the reliable use of cross correlation approach, and preventing potential errors in the arrival time difference determination of continuous AE sources.

\section{Experimental Validation of the Proposed Location Methodology}

\subsection{Experimental Design}

The experimental design includes a pipeline system made of $2.54 \mathrm{~cm}$ diameter, $0.61 \mathrm{~m}$ long polyvinyl chloride (PVC) pipes as shown in Figure 3. Orifice simulates a joint leak. The air inlet velocity is adjusted in order to have three different pressure levels measured using a pressure gauge. The leak simulation was repeated from two joint locations. Three conventional piezoelectric AE sensors, known as R6, which have $60 \mathrm{kHz}$ resonant frequency, are mounted on the pipeline structure using hot glue. A PCI-8 data acquisition system is used in this study. The data acquisition setup is $5-400 \mathrm{kHz}$ analog filter, and $35 \mathrm{~dB}$ threshold. The AE sensors are synchronized such a way that if there is a trigger on a channel (i.e. signal level is above 
threshold), the system records waveforms from all the channels. The waveform length is set up as 2 milliseconds, which allow sufficient time for the leak wave to propagate from the nearest sensor location to the next sensor location.

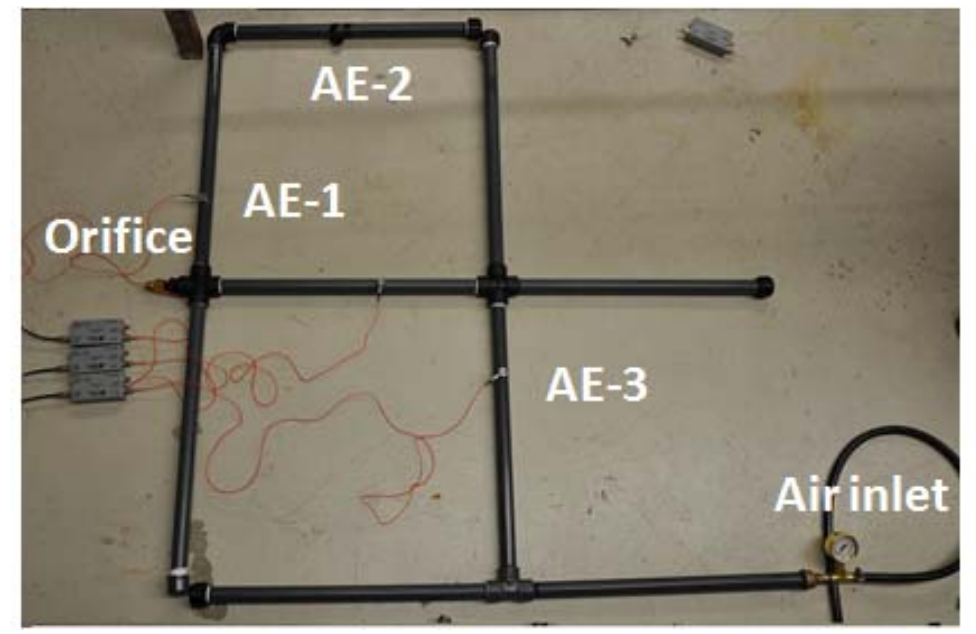

Figure 3 -Pipeline network configuration 1 for the feasibility test

\subsection{Wave Attenuation and Velocity}

Polymer is a highly attenuative material due to its high viscosity. Viscoelasticity affects the propagating elastic waves through damping the wavefront. The phase velocity $c$ for time harmonic waves is a complex number as a function of wavenumber $k$ as given by the equation [17]:

$$
c=c_{1}(k)+i c_{2}(k)
$$

The first term indicates dispersive viscoelastic waves; the second term with imaginary part indicates the amplitude loss with the increase of wave number. Achenbach [17] shows that the wave number is linearly proportional to the frequency. Therefore, high frequency waves attenuate highly in viscoelastic materials, and diminish when they confront with interfaces or connection points.

In order to demonstrate the dispersion effect on velocity and amplitude, two $0.61 \mathrm{~m}$ long pipes were connected using a coupling. A R6 sensor was used as the actuator to initiate the propagation of elastic waves. The distance between the receiving sensor and the pulsing sensor was increased gradually with $0.1 \mathrm{~m}$ increments. Figure $4 \mathrm{a}$ shows the waveform signature recorded from $0.1 \mathrm{~m}$ from the source; Figure $4 \mathrm{~b}$ shows the waveform signature recorded from $0.6 \mathrm{~m}$ (on the coupling) from the source. High frequency components of propagating elastic waves diminish with distance, and the dispersion is more indicative when the propagating waves move away from the source. Figure $4 \mathrm{c}$ shows the cross correlation plot indicating the arrival time difference of two observation locations. 

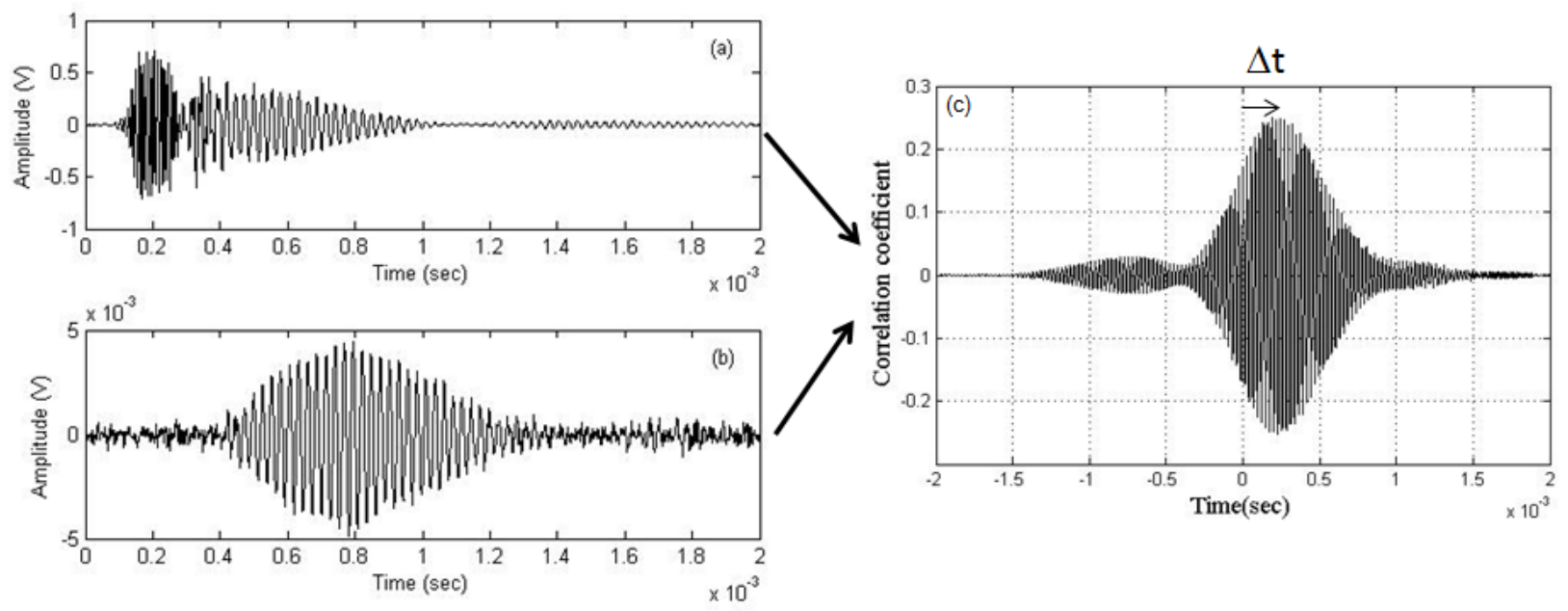

Figure 4 - Waveforms recorded at (a) $0.1 \mathrm{~m}$ from the source, (b) $0.6 \mathrm{~m}$ from the source, (c) cross correlation of two waveforms and arrival time difference as $\Delta \mathrm{t}$

The amplitudes recorded at every $0.1 \mathrm{~m}$ up to $0.6 \mathrm{~m}$ are plotted in Figure 5, which provides the attenuation curve. The slope of the linear curve fit is $0.88 \mathrm{~dB} / \mathrm{cm}$ with R square as 0.967 indicating the goodness of fit. The attenuation coefficient obtained on the $2.54 \mathrm{~cm}$ PVC pipe using $60 \mathrm{kHz}$ sensor indicates that $60 \mathrm{kHz}$ is not an appropriate frequency for detecting leakage in long PVC pipeline networks. As the attenuation coefficient decreases with frequency, lower frequency AE sensors can be selected for polymer based pipelines in order to monitor leakage with reasonable sensor spacing.

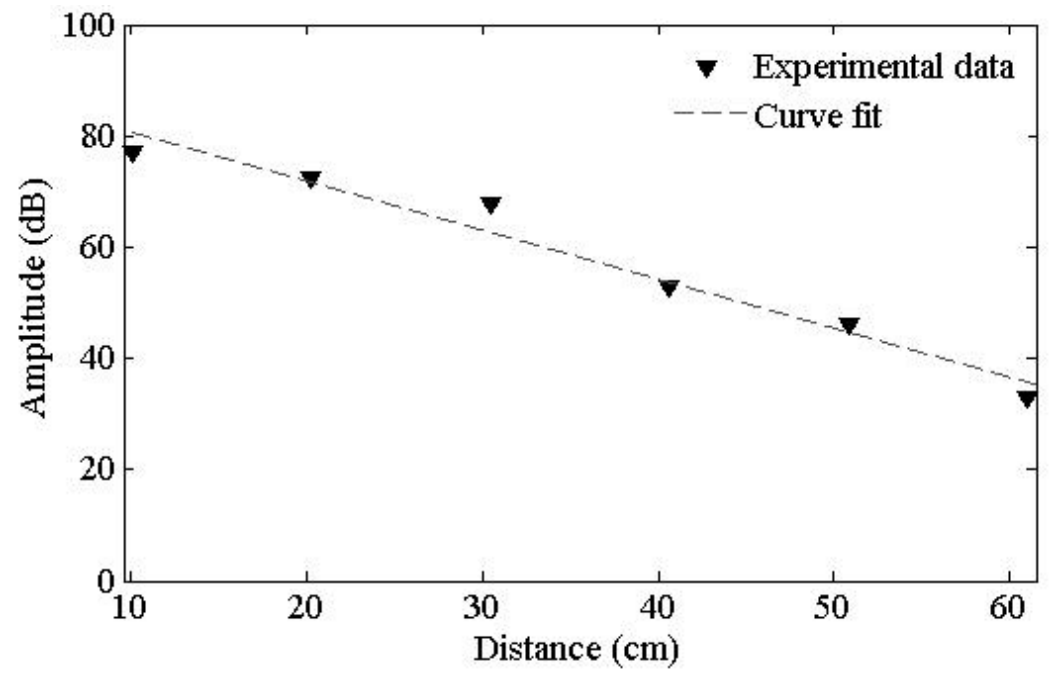

Figure 5 - Attenuation curve along $2.54 \mathrm{~cm}$ diameter PVC pipe

The arrival time differences of each source-sensor distance are calculated using the cross correlation method. The ratio of source-sensor distance to the arrival time difference provides the wave velocity as given in Table 1 . The value ' $\mathrm{j}$ ' in the table represents the reference distance in the row for applying the cross correlation. For example, the value ' $\mathrm{j}$ ' for the row corresponding to $\mathrm{R}_{0.1}$ is 0.1 so that the waveform recorded at $0.1 \mathrm{~m}$ is cross correlated with waveforms recorded at $0.2 \mathrm{~m}, 0.3 \mathrm{~m}, 0.4 \mathrm{~m}, 0.5 \mathrm{~m}$ and $0.6 \mathrm{~m}$. There are two outliers in the table as highlighted. When they are excluded from the data set, the average wave velocity is obtained as $1479.4 \mathrm{~m} / \mathrm{sec}$ with 
$636.5 \mathrm{~m} / \mathrm{sec}$ standard deviation. The standard deviation indicates the expected error in source localization due to the variance in the wave velocity caused by dispersion.

Table 1 - Velocity values using simulation and cross correlation for the calculation of the arrival time difference

\begin{tabular}{llllll}
\hline $\begin{array}{l}\text { Cross } \\
\text { correlation } \\
\text { locations }\end{array}$ & $\mathrm{R}_{\mathrm{j}+0.1}$ & $\mathrm{R}_{\mathrm{j}+0.2}$ & $\mathrm{R}_{\mathrm{j}+0.3}$ & $\mathrm{R}_{\mathrm{j}+0.4}$ & $\mathrm{R}_{\mathrm{j}+0.5}$ \\
\hline $\mathrm{R}_{0.1}$ & $1,563.1$ & $1,693.3$ & $2,005.3$ & $\mathbf{3 1 , 2 6 1 . 5}$ & $2,702.1$ \\
\hline $\mathrm{R}_{0.2}$ & $1,336.8$ & 927.9 & $1,444.5$ & $1,119.6$ & \\
\hline $\mathrm{R}_{0.3}$ & 710.5 & $1,798.2$ & $1,313.8$ & & \\
\hline $\mathrm{R}_{0.4}$ & $\mathbf{( 3 , 2 7 7 . 4 )}$ & $2,257.8$ & & & \\
\hline $\mathrm{R}_{0.5}$ & 359.0 & & & & \\
\hline
\end{tabular}

\subsection{D Source Location}

The AE sensor responses to the leak source can be chaotic, which may cause uncertainty and high probability of error in source location. The reliable source location needs more known parameters than arrival times in order to locate the leak source accurately. Due to the attenuation, the AE sensors away from the leak source detect the source with lower amplitude as compared to the AE sensor closest to the sensor (i.e. the first hit sensor). Average Signal Level (ASL) is a measure of signal amplitude detected by the $\mathrm{AE}$ sensor and is calculated by averaging signal amplitude in a defined time window. Figure 6a shows the pipeline configuration studied. PVC pipes are connected with couplings. Three AE sensors are located on three pipes in order to monitor six joints. The orifice location simulating leak is near the AE sensor 1 . The AE sensor- 2 is the second closest sensor to the leak location, and the AE sensor-3 is the furthest sensor. Figure $6 \mathrm{~b}$ shows the ASL distributions of three AE sensors due to the leak. The pressure increases between 10-20 seconds of testing, and is kept constant between 20-40 seconds of testing. Finally, the air inlet is turned off, and the pressure goes back to zero. The order of ASL values of three $\mathrm{AE}$ sensors agrees with the closeness of the sensors to the leak source. This information is used as the first input to the source location algorithm in order to identify the hit sequence.
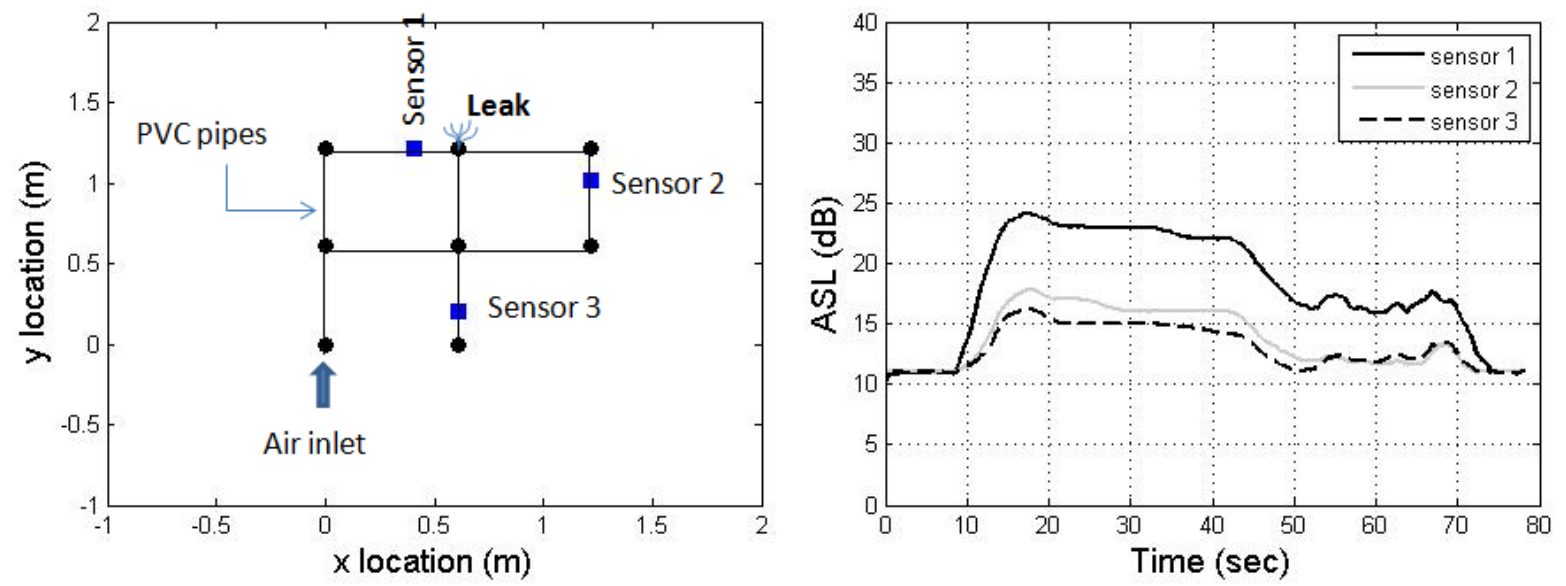

Figure 6 - (a) Pipeline network and the AE sensors for leak location-1; (b) ASL distributions of three sensors due to leak near sensor 1 under $138 \mathrm{kPa}$ pressure. 
In summary, the leak localization algorithm has the following steps:

- Identify the hit sequence based on ASL values;

- Apply the cross correlation to each sensor couple in order to determine the arrival times;

- Identify the distance between the first hit and second hit sensors, $L_{s_{1}-s_{2}}$, using the matrix $\boldsymbol{C}$ which represents the sensor-joint connectivity ( 0 if the joints are not in the neighborhood of the sensor, 1 if the joints are in the neighborhood of the sensor) and the matrices $\boldsymbol{J}$ and $\boldsymbol{S}$ which are the coordinates of joints and sensors, respectively;

- Determine the local coordinate of the source using equation 3;

- Convert the local coordinate into the global coordinate using equation 4 in order to identify the source location in multi-dimension.

Two leak locations with different pipeline configurations are studied, and the results are discussed in Section 5.4.

\subsection{Experimental Results}

The leak locations and the sensor locations are shown in Figure 7 and Figure 8. The matrices needed for the algorithm as $\boldsymbol{C}$ (the sensor-joint connectivity), $\boldsymbol{M}$ (the joint numbers at the ends of each sensor), $\boldsymbol{J}$ (the coordinates of joints) and $\boldsymbol{S}$ (the coordinates of sensors) are given in the figures. As an explanation of the matrix $\boldsymbol{C}$, for the leak location 1 (Figure 7), the first row of $\boldsymbol{C}$ matrix is 0 as there is no sensor at the pipe elements connected to the joint 1 (J1) while the first term of the third row is 1 as the $\mathrm{AE}$ sensor $1(\mathrm{AE}-1)$ is mounted on the pipe element connected to the joint 3 (J3). The matrix $\boldsymbol{J}$ indicates the joint coordinates in $-\mathrm{x}$ (the first column) and $-\mathrm{y}$ (the second column) directions. The joint 1 (J1) is considered as the origin; therefore, its coordinates are $(0,0)$ for $(\mathrm{x}, \mathrm{y})$. The matrix $\boldsymbol{M}$ indicates the joint numbers at the ends of each sensor. For example, the joints at two ends of the AE sensor 1 (AE-1) are J3 and J6; therefore, the first column values of the $\boldsymbol{M}$ matrix are 3 and 6. The matrix $\boldsymbol{S}$ is for the coordinates of sensor positions. As there are three AE sensors, and the localization is studied in $2 \mathrm{D}$, the matrix dimension is $3 \times 2$.

The located AE sources are presented as red stars in the figures. The location error for the orifice location 1(Figure 7) using $138 \mathrm{kPa}$ pressure is $37 \%$ for $-\mathrm{x}$ location and $0 \%$ for $-\mathrm{y}$ location. The errors for $207 \mathrm{kPa}$ and $276 \mathrm{kPa}$ pressures are 33\% and 23\% for $-\mathrm{x}$ location and $0 \%$ for $-\mathrm{y}$ locations, respectively. The location error for the orifice location 2 (Figure 8) using $138 \mathrm{kPa}$ pressure is $0 \%$ for $-\mathrm{x}$ location and $13 \%$ for $-\mathrm{y}$ location. The errors for $207 \mathrm{kPa}$ and $276 \mathrm{kPa}$ pressures are $0 \%$ for $-\mathrm{x}$ locations and $18 \%$ and $15 \%$ for $-\mathrm{y}$ locations, respectively. The wave velocity is selected as $1479.4 \mathrm{~m} / \mathrm{sec}$ based on the study discussed in Section 5.2. The location error for the leak location 1 is higher than the location error of the leak location 2. The AE sensor 1 for the leak location 1 is closer to the leak source, which results in less dispersive waveform as compared to the waveforms of the AE sensors 2 and 3. The AE sensors for the leak location 2 are relatively further from the leak location. The dispersion is the main cause of error in arrival time determination in this study. However, the localization error in general depends on wave velocity in addition to the identification of arrival times. The intensity of waves propagating in a material and reaching to the AE sensor due to leak depends on leak rate, pipeline pressure, geometry, material, and boundary conditions. If the leak source generates severe turbulence and material is not attenuative, the likelihood of accurate leak identification increases. 


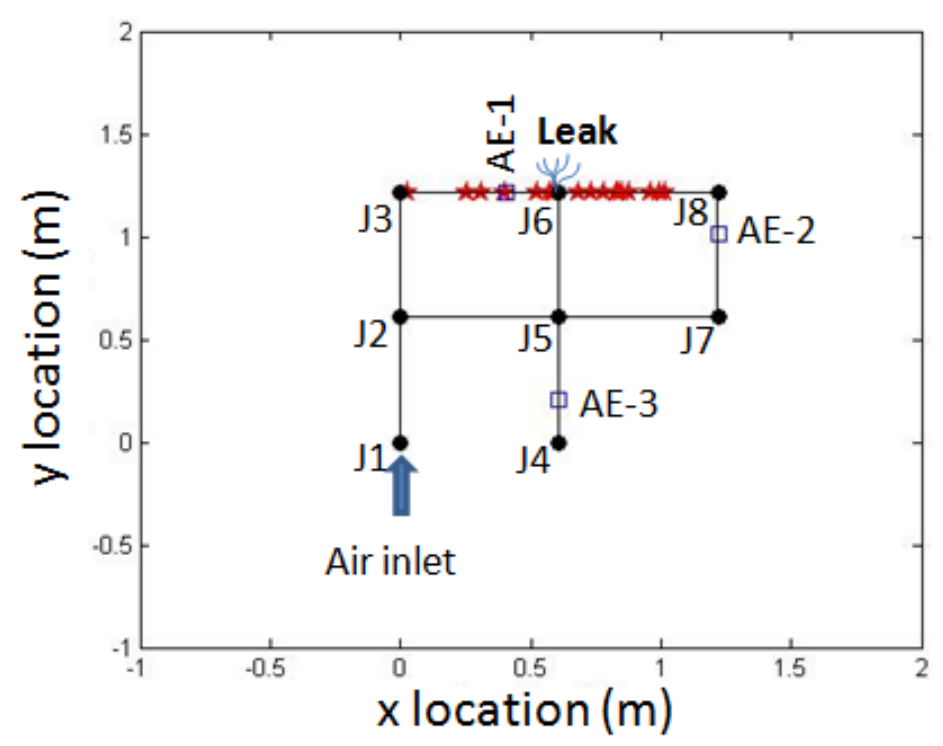

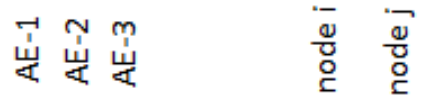

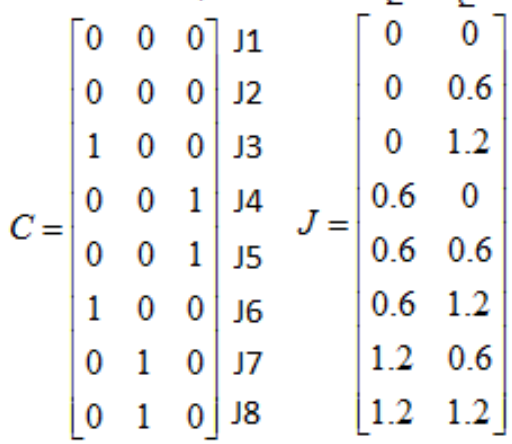

$$
\begin{aligned}
& M=\left[\begin{array}{lll}
3 & 7 & 4 \\
6 & 8 & 5
\end{array}\right] \quad S=\left[\begin{array}{cc}
0.4 & 1.2 \\
1.2 & 1 \\
0.6 & 0.2
\end{array}\right]
\end{aligned}
$$

Figure 7 - The pipeline network configuration for the leak location 1 and the corresponding matrices for the source location (units as meter for $\boldsymbol{J}$ and $\boldsymbol{S}$ matrices)

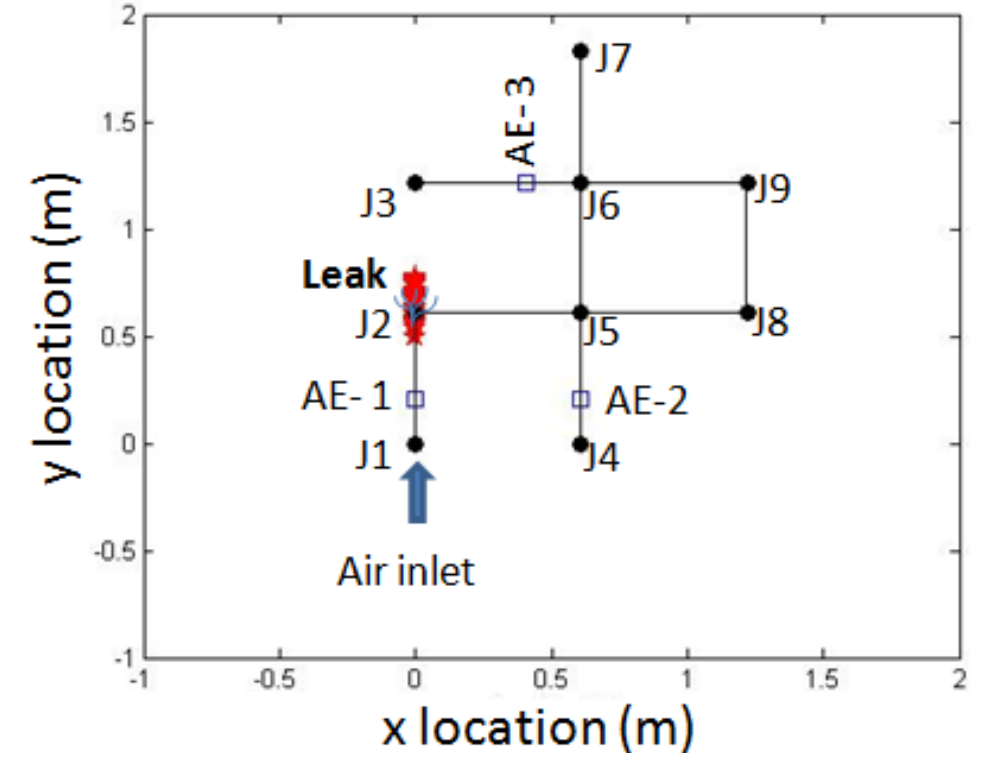

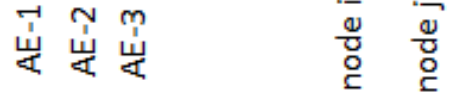

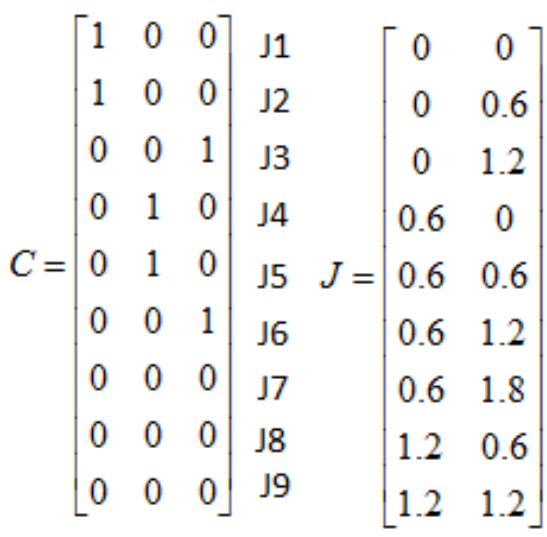

$$
\begin{aligned}
& M=\left[\begin{array}{lll}
1 & 4 & 3 \\
2 & 5 & 6
\end{array}\right] \quad S=\left[\begin{array}{cc}
0 & 0.2 \\
0.6 & 0.2 \\
0.4 & 1.2
\end{array}\right]
\end{aligned}
$$

Figure 8 - The pipeline network configuration for the leak location 2 and the corresponding matrices for the source location (units as meter for $\boldsymbol{J}$ and $\boldsymbol{S}$ matrices)

\section{Discussion and Conclusion}

This paper demonstrates that the 2D location of a leak in a pipeline network can be determined using the 1D source location algorithm integrated with geometric connectivity. As leak source generates continuous acoustic emissions which can be highly chaotic, cross correlation approach should be integrated with the hit sequence identification based on the ASL distributions of the $\mathrm{AE}$ sensors in order to reduce the error of the arrival time differences of the AE sensors. The source location algorithm presented in this study is applicable to any novel AE sensors such as 
fiber optic and MEMS. The attenuation and the wave velocity study with distance can be integrated with the location model for any kinds of pipeline materials in order to increase the reliable leakage location.

\section{Acknowledgement}

The authors would like to thank the WISEST program at the UIC for their support to the corresponding author's research. The authors acknowledge the contribution of Dr. Zhong Li during building the prototype pipelines. All opinions, findings, and conclusions are those of the authors.

\section{References}

[1] Kishawy, H.A. and Gabbar, H.A. "Review of Pipeline Integrity Management Practices,” International Journal of Pressure Vessels and Piping, 2010, Vol. 87, pp. 373-380.

[2] Rose, J.L., Cho, Y. and Avioli, M.J. "Next Generation Guided Wave Health Monitoring for Long Range Inspection of Pipes,” Journal of Loss Prevention in the Process Industries, 2009, Vol. 22, pp. 1010-1015.

[3] Elliott, J., Fletcher, R. and Wrigglesworth, M. "Seeking the Hidden Threat: Applications of a New Approach in Pipeline Leak Detection,” Abu Dhabi International Petroleum Exhibition and Conference, 3-6 November 2008, Paper No. 118070-MS.

[4] Caleyo, F., Alfonso, L. Alcantara, J. and Hallen, J.M. "On the Estimation of Failure Rates of Multiple Pipeline Systems,” Journal of Pressure Vessel Technology, 2008, Vol. 130, pp. 1-8.

[5] Shinozuka, M., Feng, M., Mosallam A. and Chou, P. “Wireless MEMS-Sensor Networks for Monitoring and Condition Assessment of Lifeline Systems,” 2007, Urban Remote Sensing Joint Event.

[6] Wan, C., Mita, A. and Kume, T. “Am Automatic Pipeline Monitoring System using Sound Information,” Structural Control and Health Monitoring, 2010, Vol.17, pp. 83-97.

[7] Higgins, M.S. and Paulson, P.O. "Fiber Optic Sensors for Acoustic Monitoring of PCCP,” 2006, ASCE Conf. Proc. for Pipeline Asset Management.

[8] Inaudi, D. and Glisic, B. "Long-Range Pipeline Monitoring by Distributed Fiber Optic Sensing,” Journal of Pressure Vessel Techology, 2010, Vol. 132, pp. 011701 1-9.

[9] ASTM E 1316 - Standard Terminology for Nondestructive Examinations.

[10] Majeed, M.A. and Murthy, C.R.L. “A Model with Nonzero Rise Time for AE Signals,” Sadhana, 2001, Vol. 25, Part 5, pp. 465-474.

[11] Ennaceur, C., Laksimi, E., Hervé, C. and Cherfaoui, M. "Monitoring Crack Growth in Pressure Vessel Steels by the Acoustic Emission Technique and the Method of Potential Difference,” International Journal of Pressure Vessels and Piping, 2006, Vol. 83, pp. 197-204.

[12] Raj, B., Mukhopadhyay, C.K. and Jayakumar, T. "Frontiers in NDE Research Nearing Maturity for Exploitation to Ensure Structural Integrity of Pressure Retaining Components,” International Journal of Pressure Vessels and Piping, 2006, Vol. 83, pp. 322-335.

[13] Miller, R.K., Pollock, A.A., Watts, D.J., Carlyle, J.M., Tafuri, A.N. and Yezzi, J.J. “A Reference Standard for the Development of Acoustic Emission Pipeline Leak Detection Techniques,” NDT\&E International, 1999, Vol. 32, pp. 1-8. 
[14] Kosel, T., Grabec, I. And Muzic, P. "Location of Acoustic Emission Sources Generated by Air Flow,” Ultrasonics, 2000, Vol. 38, pp. 824-826.

[15] Collins M.P. and Belchamber, R.M. "Acoustic Emission Source Location Using Simplex Optimization,” Journal of Acoustic Emission, 9 (4), 1989, pp. 271-276.

[16] Ozevin, D., "Geometry Based Acoustic Source Location for Spaced Frames,” International Journal of Structural Health Monitoring, September 2011, Vol. 10, No. 5, pp. 503-510.

[17] Achenbach, J.D. Wave Propagation in Elastic Solids. 1975. North-Holland.

[18] Grabec, I. "Application of Correlation Techniques for Localization of Acoustic Emission Sources,” Ultrasonics, 1978, Vol. 16, No. 3, pp. 111-115.

[19] Denbigh, P. "System Analysis \& Signal Processing: With Emphasis on The Use of Matlab," 1998, Prentice Hall, 1st Edition.

[20] Kosel, T., Grabec, I., and Kosel, F. "Intelligent Location of Two Simultaneously Active Acoustic Emission Sources: Part II,” Aircraft Engineering and Aerospace Technology: an International Journal, 2003, Vol. 75, No. 2, pp. 137-142.

[21] Gao, Y., Brennan, M.J., Joseph, P.F., Muggleton, J.M. and Hunaidi, O. “A Model of the Correlation Function of Leak Noise in Buried Plastic Pipes,” Journal of Sound and Vibration, 2004, Vol. 277, pp. 133-148.

[22] Jiao, J., He, C., Wu, B. and Fei, R. “A New Technique for Modal Acoustic Emission Pipeline Leak Location with One Sensor,” Insight, 2004, Vol. 46, No. 7, pp. 392-395. 